\title{
Building the DREAM interactome
}

\author{
Marcos RIVAS ${ }^{1,2}$, Diego VILLAR ${ }^{1,2}$, Paz GONZÁLEZ ${ }^{1,2}$, Xosé M. DOPAZO ${ }^{1,2}$, \\ Britt MELLSTROM ${ }^{1,2} \&$ José R. NARANJO ${ }^{1,2^{*}}$ \\ ${ }^{1}$ Department of Molecular and Cellular Biology, National Center of Biotechnology, Consejo Superior \\ Investigaciones Cientificas, Madrid 28049, Spain; \\ ${ }^{2}$ Centro Investigacion Biomedica En Red de Enfermedades Neurodegenerativas-Centro Nacional \\ de Biotecnologia, Madrid 28049, Spain
}

Received May 6, 2011; accepted June 7, 2011

\begin{abstract}
DREAM/calsenilin/KChIP3 is a calcium binding protein of the neuronal calcium sensor superfamily. DREAM interacts with DRE (downstream regulatory element) sites in the DNA to regulate transcription and with many proteins to exert specialized functions in different subcellular compartments. Work from different laboratories has identified a growing list of interacting proteins that constitutes the DREAM interactome. The knowledge of these interactions has greatly contributed to the understanding of the various physiological functions of DREAM.
\end{abstract}

calsenilin, KChIP3, protein-protein interaction

Citation: $\quad$ Rivas M, Villar D, González P, et al. Building the DREAM interactome. Sci China Life Sci, 2011, 54: 786-792, doi: 10.1007/s11427-011-4196-4

\section{DREAM, a multifunctional protein}

DREAM (Antagonist Modulator of DRE sites) was cloned in a functional screening of a cDNA library from human striatum as a transcriptional repressor able to form tetramers and bind to the DRE site in the proximal promoter of the human prodynorphin gene $[1,2]$. DREAM is expressed in brain, thyroid gland, immune system, heart and gonads and is one of four highly conserved members of the KChIP (potassium channel interacting protein) subfamily of neuronal calcium sensors $[3,4]$.

DREAM, also known as calsenilin [5] or KChIP3 [3], is a calcium binding protein that contains three functional EF-hand motifs (Figure 1) and interacts with DRE (downstream regulatory element) sites in the DNA and with many proteins to exert specialized functions in different subcellular compartments [6]. Binding of calcium induces a structural change in DREAM that precludes binding to DNA and modifies the interaction with some proteins but

*Corresponding author (email: naranjo@cnb.csic.es) not with others. For instance, $\mathrm{Ca}^{2+}$-bound DREAM does not interact with CREB [7] or with the PSD-95 protein [8], while the interaction of DREAM with presenilins [5], with potassium channels of the Kv4 class [3] or with the TSH receptor [9], is not affected by calcium.

A key property of DREAM is the ability to form homomers or heterotetramers with other KChIP proteins, which results in different affinities for DNA binding to DRE sites proximal to the transcription start site, leading to transcriptional repression of target genes $[2,4,10,11]$. The DRE sequence was defined as the element responsible for basal and induced expression of the prodynorphin gene, encoding a protein involved in nociception as well as in learning and memory $[12,13]$. More recent studies, however, have shown that many other genes also contain DRE sites and are regulated by DREAM. For instance, DRE sites contribute to basal and induced expression of a number of immediate early transcription factors including c-fos, c-jun and ICER $[2,4]$. In addition to its regulation by calcium, binding of DREAM to DNA is hampered by its interaction with phosphoCREM [14], while interaction with other transcription 


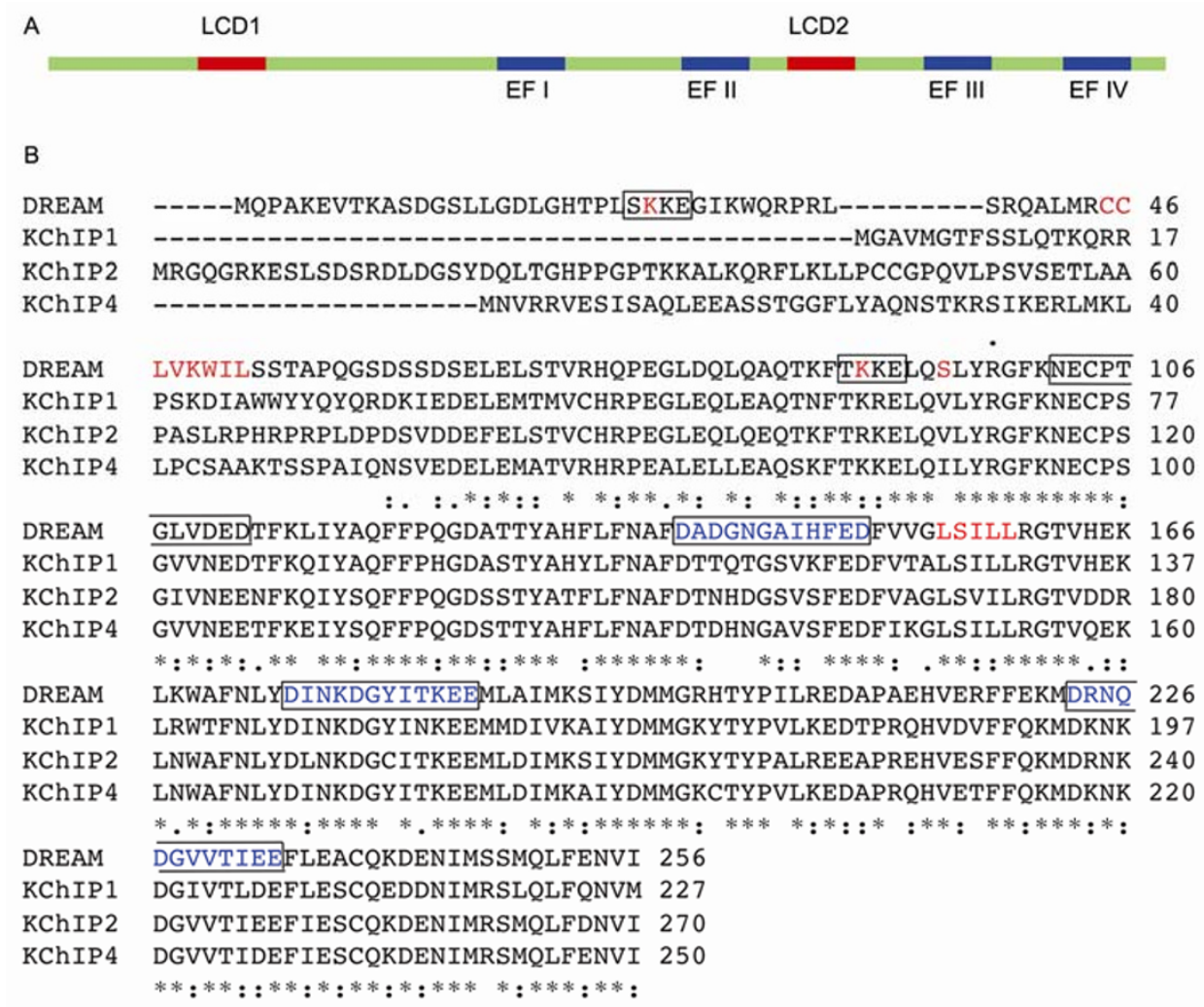

Figure 1 The DREAM protein and alignment with other KChIPs. A, Schematic representation of the DREAM protein showing the LCD and EF-hand motifs. B, Sequence alignment of DREAM and KChIP family members. The human sequences for DREAM (Acc. Q9Y2W7), KChIP 1 (Q9NZI2), KChIP 2 (Q9NS61) and KChIP 4 (Q6PIL6) were aligned. Asterisk (*) denotes identical residues, colon (:) conservative changes and period (·) related substitutions. Sumoylation sites are boxed showing the sumoylated lysine in red. EF-hand motifs are also boxed, the three functional hands depicted in blue. LCD motifs and other amino acids important for function such as cysteins at positions 45 and 46, and the phosphorylation site at serine 95 , are shown in red.

factors like CREB, TTF-1 or CtBP2 allows DREAM to regulate transcription of genes that do not contain DRE sites (Figure 2) $[7,15,16]$. Outside the nucleus, DREAM regulates many cellular functions through selective interactions with an increasing number of proteins that constitute the DREAM interactome and underscore DREAM multifunctional properties.

\section{Defining the DREAM interactome}

DREAM plays an important role in cell physiology through the interaction with specific proteins in different subcellular compartments and in various cell types. In fact, and independently to its discovery as a calcium-regulated repressor, DREAM/calsenilin was identified based on its interaction with the C-terminal fragment of presenilin-2 in a yeast two-hybrid assay [5]. The interaction was found to be insensitive to $\mathrm{Ca}^{2+}$, and as a result, DREAM was proposed to enhance presenilin-induced calcium release from the ER to the cytosol [17] and may control $\gamma$-secretase activity and A $\beta$ aggregation in neurons [18]. The role of DREAM/calsenilin in the processing of the amyloid precursor protein, however, remains controversial and different groups have published ambiguous results, including reduced levels of $\mathrm{A} \beta$ peptides in DREAM/calsenilin knock-out mice [18], increased level of DREAM/calsenilin in AD (Alzheimer's disease) brains [19] and in neuronal cultures after exposure to $A \beta 42$ [20] or unchanged level in a transgenic mouse model of AD [21].

Also applying the yeast two-hybrid approach, other $\mathrm{Ca}^{2+}$-independent interactions have been described. Using the N-terminal region of the $\mathrm{Kv} 4$ potassium channel as bait, three of the four members of the KChIP family, of which DREAM is KChIP3, were found [3]. Binding of DREAM/ $\mathrm{KChIP} 3$ to the Kv4 channel occurs in the endoplasmic reticulum and, upon binding, DREAM facilitates intracellular trafficking and membrane expression of the channel [3]. In addition, DREAM regulates the gating of the Kv4 channel in the membrane, slowing inactivation and accelerating the recovery to the functional state. Interestingly, although the binding is insensitive to $\mathrm{Ca}^{2+}$, regulation of the gating needs the integrity of the EF-hands in the DREAM protein [3]. In another yeast two-hybrid assay, using the N-terminal of DREAM as bait the interactions with the transcriptional corepressor C-terminal binding protein 2 (CtBP2) and its homolog CtBP1 were identified [16]. Interestingly, histone deacetylase (HDAC) protein and activity was found to be associated with the DREAM-CtBP immunocomplex, sug- 
A

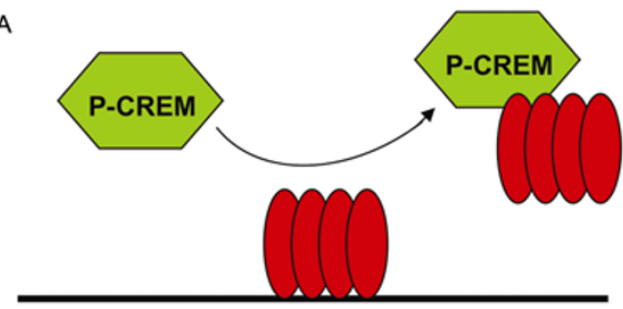

B

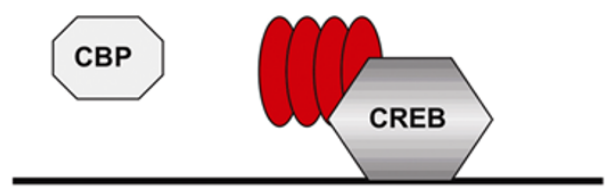

C

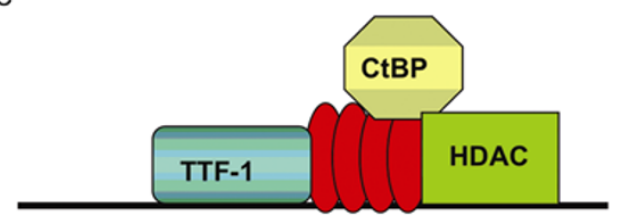

Figure 2 Schematic models of the DRE-dependent and DRE-independent modulation of the transcriptional activity of DREAM. A, Binding of DREAM to DRE sites is modulated by the interaction with phosphoCREM, which releases DREAM from the DNA preventing DREAM-mediated transcriptional repression. B, DREAM binds to CREB and prevents the interaction between CREB and its coactivator CBP, thus blocking CREB-mediated transcriptional activity. C, DREAM forms transcriptional regulatory complexes through its binding to other transcription factors (TTF-1), corepressors (CtBP) and HDACs.

gesting a mechanism by which DREAM-CtBP may act to repress transcription. Surprisingly, the DREAM-CtBP interaction was also detected in synaptic vesicles, suggesting that outside the nucleus the DREAM-CtBP interaction may play a role in the maintenance, release or trafficking of these vesicles [16]. Also the $\mathrm{Ca}^{2+}$-independent interaction of DREAM and KChIP-4 with the galactosyltransferase GalT2 was detected by yeast two-hybrid screening using the $\mathrm{N}$-terminal domain of GalT2 as bait [22]. GalT2 is a Golgiresident type II membrane protein that participates in the synthesis of glycosphingolipids. Cotransfection experiments showed that DREAM and GalT2 mutually affect their subcellular distribution. Thus, in the presence of GalT2 the diffuse distribution of DREAM partially changes, showing a tendency to form clustered structures in the perinuclear region. Interestingly, GalT2 also affects DREAM stability, accelerating its proteolysis by the proteosome. Conversely, DREAM leads to redistribution of GalT2, as well as two other galactosyltransferases (SialT2 and GalNAcT), from the Golgi to the ER. These data suggested that DREAM might be involved in the trafficking of Golgi glycosyltransferases [22].

\section{The LCD-dependent interactome}

Characterization of the DREAM interactome was initially based on results from the yeast two-hybrid assays mentioned above and on data from functional analyses of DREAM. Three examples illustrate this second source of information, the interactions with CREM, CREB and the vitamin D receptor. The interaction with CREM was discovered during studies on the regulation of DREAM DNA binding by cAMP [1,14]. These characterized the highaffinity interaction between phosphoCREM and DREAM, which was found to be mediated by two leucine-, charged residue-rich domains (LCDs) in the DREAM protein, hereafter named LCD1 and LCD2 (Figure 1). Two LCDs in CREM, one in the N-terminal domain and one in the bZIP region in the $\mathrm{C}$-terminal domain, are also needed. The interaction is facilitated by the cAMP-dependent phosphorylation of CREM at serine 68, in turn blocking binding of DREAM to the DRE site in the target gene prodynorphin and leading to derepression of its expression [14]. Based on the high sequence homology between CREM and CREB, the role of a putative LCD in CREB was investigated and, in fact, this was found to be equivalent to the CREM C-terminal LCD. Importantly, this LCD is located in the KID domain of CREB, which mediates interaction with $\mathrm{CBP} / \mathrm{p} 300$ allowing HAT recruitment and transactivation of CRE sites [23]. The interaction involves LCD1 in DREAM and is $\mathrm{Ca}^{2+}$-dependent. Consequently, $\mathrm{Ca}^{2+}$-insensitive DREAM mutants permanently occupy the KID domain, blocking the CREB-CBP interaction and CRE-dependent gene expression [7].

LCD domains were originally identified as responsible for the interaction between hormone nuclear receptors and the transcriptional machinery [24]. Thus, we searched for an LCD-based functional interaction between DREAM and nuclear receptor-dependent transcription. We disclosed that DREAM enhances basal and ligand-dependent activation of promoters containing vitamin $\mathrm{D}$ and retinoic acid response elements (VDREs and RAREs). The promoter activation requires both LCDs in DREAM and is enhanced in the presence of $\mathrm{Ca}^{2+}$ [25]. Chromatin immunoprecipitation using vitamin D- or retinoic acid-treated cells demonstrated the presence of DREAM in nuclear complexes bound to regulatory regions containing VDRE and RAREs [25] and pull-down assay has confirmed the direct interaction between DREAM and vitamin D receptors [26]. Taken together, these interactions show that, in the presence of $\mathrm{Ca}^{2+}$, DREAM can function as a transcriptional activator of promoters containing CRE sites or binding sites for nuclear receptors.

\section{The thyroid-specific DREAM interactome}

Analysis of the functional role of DREAM in the thyroid 
gland showed that DREAM interacts with the N-terminal region of TTF-1 and impairs the transcriptional activity of its main transactivating domain to downregulate thyroglobulin gene expression [15]. Chromatin immunoprecipitation experiments showed that DREAM binds to the thyroglobulin promoter and promoter deletion analysis identified the binding of DREAM to a region overlapping the binding site for TTF-1 and Pax-8 in the thyroglobulin promoter. These results support the idea that DREAM may form transcriptional regulatory complexes with other partners on the promoters of their target genes [15]. Interestingly, the interaction between DREAM and TTF-1 does not involve the LCD domains of DREAM.

Furthermore, working with thyroid follicular cells, we found that DREAM interacts with the C-terminal intracellular part of TSHR regulating its membrane expression and promoting its binding to the Ga subunit. As a result, DREAM over-expression led to enhanced activation of TSHR activity with increased induction of the cAMP signalling cascade [9]. Modulation of TSHR protein levels and increased receptor activity were achieved through independent mechanisms. Thus, a DREAM-derived peptide containing amino acids 43-62 was able to activate the receptor without affecting its protein level. Accordingly, membrane stabilization of TSHR by the $\mathrm{Ca}^{2+}$-binding chaperone calreticulin did not induce the cAMP cascade. Importantly, interaction with THSR requires the N-terminal region, amino acids 1-90, of DREAM, which is not present in the other members of the KChIP family. Thus, the effect of DREAM on TSHR is not reproduced by KChIP-2, which is also expressed in the thyroid gland $[9,15]$. Therefore, despite the great sequence homology and redundancy in some activities among the different KChIP proteins $[3,4]$, the N-terminal part determines specific functions for DREAM.

\section{$5 \mathrm{Ca}^{2+}$-dependent interactions revealed by yeast two-hybrid}

The interactions found as a result of functional studies turned out in most of the cases to be $\mathrm{Ca}^{2+}$-dependent, while those discovered using the yeast two-hybrid assay were not sensitive to calcium. In this situation, it was reasonable to think that perhaps the high calcium concentration in yeast cytosol precluded the calcium-sensitive interactions between DREAM and bait proteins. To overcome this potential technical bias, we designed a yeast two-hybrid assay using as bait a $\mathrm{Ca}^{2+}$-insensitive DREAM mutant, with mutations in each one of the three functional EF-hands. Results from this screening confirmed the hypothesis and allowed us to describe several new interactions that were in all cases sensitive to the presence of calcium. Three of these interactions, namely with GRK, Ubc9 and Prdx3, have been recently characterized at the molecular level, providing new insights in the functionality of the DREAM protein.
Since the interactions between G-coupled receptor kinases (GRK) and several other neuronal calcium sensors had been already reported [27], the interaction between DREAM and GRK6 and GRK2 kinases observed was somehow a predictable result [28]. We found that the interaction with GRK6 and GRK2 results in the phosphorylation of DREAM at Ser95. The phosphorylation does not modify the repressor activity of DREAM, but dramatically influences the trafficking of Kv4 potassium channels from the ER to the cell membrane [28]. Thus, mutation of Ser95 to aspartic acid blocked DREAM-mediated membrane expression of Kv4.2 potassium channels without affecting channel tetramerization. Furthermore, treatment with the calcineurin inhibitors, FK506 and cyclosporin A, also blocked DREAM-mediated Kv4.2 channel trafficking suggesting that calcineurin participates in the dephosphorylation of Ser95 phosphoDREAM. Thus, both phosphorylation and dephosphorylation of DREAM at Ser95 are tightly regulated in a $\mathrm{Ca}^{2+}$-dependent manner and so is the activity of DREAM on Kv4.2 channel function [28].

The interaction with the SUMO-conjugating enzyme Ubc9 led to the identification of DREAM as a target for sumoylation. Single K-to-R mutations at positions K26 and K90 prevent in vitro sumoylation of recombinant DREAM and reduce nuclear localization of DREAM [29]. These results suggested that sumoylation regulates the nuclear localization of DREAM and thereby determines its transcriptional repressor activity. Indeed, it was shown that in PC12 cells, sumoylated DREAM is present exclusively in the nucleus, and accordingly, in fully differentiated trigeminal neurons, DREAM and SUMO-1 colocalize in nuclear domains associated with transcription [29]. Sequence analysis of the four members of the KChIP family (Figure 1) shows that the sumoylation consensus site K26, located in the DREAM-specific N-terminal region, is not present in the other three members, while the sumoylation site at K90 is conserved in KChIP 1 and 2 but not in KChIP4. Thus, sumoylation of other KChIP family members may occur but is presently unknown. Whether sumoylation alters the interaction of DREAM with other nucleoproteins and whether DREAM affinity or specificity for DRE sites is affected by sumoylation remains to be analyzed.

Third, the yeast two-hybrid assay with mutant DREAM as bait disclosed the interaction with peroxiredoxin 3 (Prdx3), an antioxidant enzyme of the thioredoxin system that protects neurons from oxidative damage [30]. The DREAM-Prdx3 interaction is greatly reduced in the presence of calcium and is blocked in the presence of DTT, suggesting that DREAM preferentially interacts with Prdx3 under oxidizing conditions. The interaction is specific for $\operatorname{Prdx} 3$ and was not observed for other members of the Prdx family, such as the nuclear localized Prdx5. The functional relevance of this interaction is intriguing, since $\operatorname{Prdx} 3$ is a mitochondrial protein, not located in the cytosol or in the nucleus, while mitochondrial localization for DREAM has 
so far not been reported. Therefore, whether this interaction takes place within the mitochondria or in the cytosol following the release of mitochondrial factors during oxidative stress remains an open question. The DREAM-Prdx3 interaction led us to demonstrate that DREAM plays a neuroprotective role in response to oxidative stress. Moreover, it served to disclose that both DNA-binding and oligomerization activity of DREAM are modulated by the redox state of two cysteine residues located at positions 45 and 46. Mutation of Cys to Ser in the DREAM-C45,46S mutant was used to investigate the influence of the redox state on DREAM activity. Cys to Ser mutations mimic a permanent reduced form of cysteins and prevent the formation of either intra- or intermolecular disulfide bonds. As a result, the DREAMC45,46S mutant showed greater dimer formation capacity and stronger repressor effect on DRE-dependent transcription [30]. Since these two residues are immediately preceding LCD1, it is likely that their redox state may also affect the interaction with other DREAM partners such as CREM or CREB. Moreover, KChIP2 also contains double Cys residues in equivalent positions, while KChIP1 and 4 contains a single Cys residue (Figure 1). This suggests that redox signaling might favor the formation of specific DREAM/KChIP homo- and hetero-oligomers to elicit specific functions.

\section{Recent additions to the membrane-specific DREAM interactome}

In addition to the mentioned interactions of DREAM with Kv4 channels and TSHR, several recent studies are uncovering novel interactions that point to an expanded role of KChIP proteins in the regulation of membrane-bound proteins. While studying the reduction of NMDA-mediated currents in transgenic neurons expressing a $\mathrm{Ca}^{2+}$-insensitive DREAM mutant, we identified the $\mathrm{Ca}^{2+}$-dependent interaction with PSD-95 [8]. We proposed that this interaction could compete the PSD-95/src interaction, in turn precluding NR2B Tyr-phosphorylation and NMDA receptor full activity [8]. Interestingly, an independent study confirmed the reduction of NMDA-mediated currents by DREAM, though in this case the mechanism involved a $\mathrm{Ca}^{2+}$-dependent interaction between the N-terminal region of DREAM and the NR1 subunit of the NMDA receptor [31]. Moreover, it was shown that wild-type DREAM and DREAM N-terminal peptides negatively regulate NMDA currents and reduce NMDA-induced cytotoxicity [31]. Together, these studies propose that DREAM impinges on NMDA-mediated currents at multiple levels. Since the N-terminal residues 1-50 of DREAM are not conserved in the other KChIP proteins, these results also indicate that the interaction and modulation of the NMDA receptor is DREAM specific and not shared by other KChIPs.

Two recent reports further implicate DREAM in regula- tion of membrane conductance. One report demonstrates that DREAM forms a signaling complex with Kv4 and voltage-dependent T-type calcium channels (Cav3) in stellate cells [32]. Electrophysiological measurements propose that T-type channels efficiently couple calcium influx to DREAM/KChIP3 to modulate Kv4 function, establishing DREAM as a physiological calcium sensor for the Kv4 channel in neurons. A second work using $\mathrm{KChIP}^{-/-}$cardiac myocytes has shown that KChIP2 directly interacts with voltage-dependent L-type calcium channels (Cav1.2) augmenting their current amplitude [33]. Whether other KChIPs can also interact with $\mathrm{Ca}^{2+}$ channels and the physiological significance in different tissues remains to be investigated. Nevertheless, these reports collectively point to DREAM and other KChIP proteins as key modulators of membrane conductance through the interaction with multiple cationic channels.

\section{New approaches to uncover the DREAM in- teractome}

With the exception of the interaction with the transcriptional corepressor $\mathrm{CtBP} 2$, all positive interactions in yeast twohybrid assays correspond to cytosolic or membrane-bound proteins. This may be due to a low representation of nucleoprotein-encoding clones in yeast libraries or, alternatively, it may be related to the complexity of these interactions, which are not favored in the yeast environment. For instance, although the interactions of CREM or CREB with DREAM are strong and reliable, no yeast clones encoding CREM or CREB were obtained. Of note, these interactions were also absent in a recent high-throughput yeast two-hybrid screening aiming to a systematic mapping of proteinprotein interactions, despite DREAM ORFs being represented in the employed library [34]. To overcome these potential technical drawbacks, other methodological approaches could be attractive alternatives, including affinity pull-down techniques, high-density protein microarrays and stable isotopic labeling coupled to immunoprecipitation, liquid chromatography and mass spectrometry analysis.

A representative example of one of these approaches is the search for direct protein-protein interactions between Taiwan cobra cardiotoxin3 (CTX3) and KChIPs, using affinity pull-down assays and chemical cross-linking [35]. Cardio toxins (Cuts) form a group of polypeptides present in the elapid family of snakes. They are known to have $\mathrm{Ca}^{2+}$-dependent cellular effects and a diversity of pharmacological functions, including hemolysis, cytotoxicity, and depolarization of muscles. All KChIPs interact with CTX3 albeit detailed characterization of the interaction was carried out only for KChIP-1. Binding of CTX3 to KChIP1 required the integrity of EF-hands 3 and 4 of KChIP1, although the interaction was found to be $\mathrm{Ca}^{2+}$-independent. KChIP-1 was able to interact simultaneously with CTX3 
Table 1 DREAM interacting proteins

\begin{tabular}{|c|c|c|c|c|}
\hline Gene & $\mathrm{Ca}^{2+}$ & Compartment & Function & References \\
\hline CREM & + & nucleus & transactivation & Ledo [14] \\
\hline CREB & + & nucleus & transactivation & Ledo [7] \\
\hline presenilin & - & cytosol & $\mathrm{ER} \mathrm{Ca}^{2+}$ release & Buxbaum [5] \\
\hline $\mathrm{Kv} 4$ & - & ER & trafficking/gating & An [3] \\
\hline TSHR & - & membrane & receptor modulation & Rivas [9] \\
\hline GRK & + & cytosol & trafficking & Ruiz-Gomez [28] \\
\hline $\operatorname{Prdx} 3$ & + & n.d. ${ }^{\text {a) }}$ & n.d. & Rivas [30] \\
\hline PSD95 & + & cytosol & NMDAR activity & $\mathrm{Wu}[8]$ \\
\hline Cav T-type & + & membrane & receptor modulation & Anderson [32] \\
\hline Cav L-type & + & membrane & receptor modulation & Thomsen [33] \\
\hline GalT2 & - & Golgi & trafficking/proteolysis & Quintero [22] \\
\hline $\mathrm{CtBP}$ & - & synaptic vesicles & transactivation & Zaidi [16] \\
\hline CTX3 & - & membrane & Kv modulation & Lin [35] \\
\hline NMDR1 & + & membrane & current regulation & Zhang [31] \\
\hline
\end{tabular}

a) n.d. = not determined.

and the N-terminal fragment of the Kv4.2 channel. Interestingly, the interaction between KChIP-1 and the channel was enhanced in the presence of CTX3, which suggests that CTX3 might regulate the electrophysiological properties of $\mathrm{Kv}$ channels. Given the high sequence homology and functional redundancy of the KChIP proteins in terms of the regulation of $\mathrm{Kv} 4$ channels, we speculate that KChIP3/ DREAM would behave similarly.

\section{Conclusion}

The list of DREAM interacting proteins (Table 1) has grown considerably in the last few years. The present inventory, far from complete, awaits exciting additions that, for instance, should complete the nuclear DREAM interactome by identifying elements of the basic transcriptional machinery that interact with DREAM and define its transcriptional repressor activity. New interactions should also be expected at the cell membrane. One reason for this is that DREAM might share in part the interactome of other neuronal calcium sensors [36], as shown for GRKs, and if so, many interactions of specific functional significance could be disclosed. Moreover, future studies of the enhanced coupling between TSHR and G proteins upon DREAM binding to the receptor may identify new interactions mediating changes in signaling, receptor trafficking and receptor stabilization. Taken together, the growing DREAM interactome highlights the diverse roles of DREAM as a multifunctional protein with specific roles in different cell compartments.

This work was supported by grants from Ministerio Ciencia e Innovacion (Grant Nos. SAF2007-62449 to José R. Naranjo, SAF2005-04682 and SAF2008-03469 to Britt Mellstrom), CIBERNED to Britt Mellstrom and José R. Naranjo and from Comunidad Autonoma de Madrid, Fundación La Caixa (Grant No. BM04-167-0) and the EU 6th Framework Program (NeuroNE) to José R. Naranjo.
1 Carrion A M, Mellstrom B, Naranjo J R. Protein kinase A-dependent derepression of the human prodynorphin gene via differential binding to an intragenic silencer element. Mol Cell Biol, 1998, 18: 69216929

2 Carrion A M, Link W A, Ledo F, et al. DREAM is a $\mathrm{Ca}^{2+}$-regulated transcriptional repressor. Nature, 1999, 398: 80-84

3 An W F, Bowlby M R, Betty M, et al. Modulation of A-type potassium channels by a family of calcium sensors. Nature, 2000, 403: 553-556

4 Link W A, Ledo F, Torres B, et al. Day-night changes in downstream regulatory element antagonist modulator/potassium channel interacting protein activity contribute to circadian gene expression in pineal gland. J Neurosci, 2004, 24: 5346-5355

5 Buxbaum J D, Choi E K, Luo Y, et al. Calsenilin: a calcium-binding protein that interacts with the presenilins and regulates the levels of a presenilin fragment. Nat Med, 1998, 4: 1177-1181

6 Mellstrom B, Savignac M, Gomez-Villafuertes R, et al. $\mathrm{Ca}^{2+}$-operated transcriptional networks: molecular mechanisms and in vivo models. Physiol Rev, 2008, 88: 421-449

7 Ledo F, Kremer L, Mellstrom B, et al. $\mathrm{Ca}^{2+}$-dependent block of CREB-CBP transcription by repressor DREAM. EMBO J, 2002, 21: 4583-4592

8 Wu L J, Mellstrom B, Wang H, et al. DREAM (Downstream Regulatory Element Antagonist Modulator) contributes to synaptic depression and contextual fear memory. Mol Brain, 2010, 3: 3

9 Rivas M, Mellstrom B, Torres B, et al. The DREAM protein is associated with thyroid enlargement and nodular development. Mol Endocrinol, 2009, 23: 862-870

10 Craig T A, Benson L M, Venyaminov S Y, et al. The metal-binding properties of DREAM: evidence for calcium-mediated changes in DREAM structure. J Biol Chem, 2002, 277: 10955-10966

11 Osawa M, Tong K I, Lilliehook C, et al. Calcium-regulated DNA binding and oligomerization of the neuronal calcium-sensing protein, calsenilin/DREAM/KChIP3. J Biol Chem, 2001, 276: 41005-41013

12 Naranjo J R, Mellstrom B, Achaval M, et al. Molecular pathways of pain: Fos/Jun-mediated activation of a noncanonical AP-1 site in the prodynorphin gene. Neuron, 1991, 6: 607-617

13 Wagner J J, Terman G W, Chavkin C. Endogenous dynorphins inhibit excitatory neurotransmission and block LTP induction in the hippocampus. Nature, 1993, 363: 451-454

14 Ledo F, Carrion A M, Link W A, et al. DREAM-alphaCREM interaction via leucine-charged domains derepresses downstream regulatory element-dependent transcription. Mol Cell Biol, 2000, 20: 9120-9126

15 Rivas M, Mellstrom B, Naranjo J R, et al. Transcriptional repressor 
DREAM interacts with thyroid transcription factor-1 and regulates thyroglobulin gene expression. J Biol Chem, 2004, 279: 33114 33122

16 Zaidi N F, Kuplast K G, Washicosky K J, et al. Calsenilin interacts with transcriptional co-repressor C-terminal binding protein(s). J Neurochem, 2006, 98: 1290-1301

17 Leissring M A, Yamasaki T R, Wasco W, et al. Calsenilin reverses presenilin-mediated enhancement of calcium signaling. Proc Natl Acad Sci USA, 2000, 97: 8590-8593

18 Lilliehook C, Bozdagi O, Yao J, et al. Altered Abeta formation and long-term potentiation in a calsenilin knock-out. J Neurosci, 2003, 23: 9097-9106

19 Jo D G, Lee J Y, Hong Y M, et al. Induction of pro-apoptotic calsenilin/DREAM/KChIP3 in Alzheimer's disease and cultured neurons after amyloid-beta exposure. J Neurochem, 2004, 88: 604611

20 Jin $\mathrm{J} \mathrm{K}$, Choi J K, Wasco W, et al. Expression of calsenilin in neurons and astrocytes in the Alzheimer's disease brain. Neuroreport, 2005, 16: 451-455

21 Smith I F, Hitt B, Green K N, et al. Enhanced caffeine-induced $\mathrm{Ca}^{2+}$ release in the $3 \times \mathrm{Tg}-\mathrm{AD}$ mouse model of Alzheimer's disease. J Neurochem, 2005, 94: 1711-1718

22 Quintero C A, Valdez-Taubas J, Ferrari M L, et al. Calsenilin and CALP interact with the cytoplasmic tail of UDP-Gal:GA2/GM2/GD2 beta-1,3-galactosyltransferase. Biochem J, 2008, 412: 19-26

23 Parker D, Ferreri K, Nakajima T, et al. Phosphorylation of CREB at Ser-133 induces complex formation with CREB-binding protein via a direct mechanism. Mol Cell Biol, 1996, 16: 694-703

24 Le Douarin B, Nielsen A L, Garnier J M, et al. A possible involvement of TIF1 alpha and TIF1 beta in the epigenetic control of transcription by nuclear receptors. EMBO J, 1996, 15: 6701-6715

25 Scsucova S, Palacios D, Savignac M, et al. The repressor DREAM acts as a transcriptional activator on Vitamin D and retinoic acid response elements. Nucleic Acids Res, 2005, 33: 2269-2279

26 Lusin J D, Vanarotti M, Li C, et al. NMR structure of DREAM: Implications for $\mathrm{Ca}(2+)$-dependent DNA binding and protein dimerization. Biochemistry, 2008, 47: 2252-2264

27 Iacovelli L, Sallese M, Mariggio S, et al. Regulation of G-proteincoupled receptor kinase subtypes by calcium sensor proteins. FASEB J, 1999, 13: 1-8

28 Ruiz-Gomez A, Mellstrom B, Tornero D, et al. G protein-coupled receptor kinase 2-mediated phosphorylation of downstream regulatory element antagonist modulator regulates membrane trafficking of Kv4.2 potassium channel. J Biol Chem, 2007, 282: 1205-1215

29 Palczewska M, Casafont I, Ghimire K, et al. Sumoylation regulates nuclear localization of repressor DREAM. Biochim Biophys Acta, 2010, 1813: 1050-1058

30 Rivas M, Aurrekoetxea K, Mellstrom B, et al. Redox signaling regulates transcriptional activity of the $\mathrm{Ca}(2+)$-dependent repressor DREAM. Antioxid Redox Signal, 2010, 14: 1237-1243

31 Zhang Y, Su P, Liang P, et al. The DREAM protein negatively regulates the NMDA receptor through interaction with the NR1 subunit. J Neurosci, 2010, 30: 7575-7586

32 Anderson D, Mehaffey W H, Iftinca M, et al. Regulation of neuronal activity by Cav3-Kv4 channel signaling complexes. Nat Neurosci, 2010, 13: 333-337

33 Thomsen M B, Wang C, Ozgen N, et al. Accessory subunit KChIP2 modulates the cardiac L-type calcium current. Circ Res, 2009, 104: $1382-1389$

34 Rual J F, Venkatesan K, Hao T, et al. Towards a proteome-scale map of the human protein-protein interaction network. Nature, 2005, 437: $1173-1178$

35 Lin $\mathrm{Y} \mathrm{L}, \mathrm{Wu} \mathrm{P} F$, Wu T T, et al. KChIP3: a binding protein for Taiwan banded krait beta-bungarotoxin. Toxicon, 2006, 47: 265-270

36 Burgoyne R D. Neuronal calcium sensor proteins: generating diversity in neuronal $\mathrm{Ca}^{2+}$ signalling. Nat Rev Neurosci, 2007, 8: 182-193

Open Access This article is distributed under the terms of the Creative Commons Attribution License which permits any use, distribution, and reproduction in any medium, provided the original author(s) and source are credited. 